\title{
ESTUDO DA ADESÃO DO ÓXIDO DE GRAFENO EM UM TECIDO DE POLIAMIDA AROMÁTICA POR TRATAMENTO TÉRMICO
}

\author{
Anderson Oliveira da Silva ${ }^{1}$ \\ Andreza Menezes Lima ${ }^{2}$ \\ Géssica Seara Faria ${ }^{3}$ \\ Karollyne Gomes de Castro Monsores ${ }^{4}$ \\ Maria de Fátima Vieira Marques ${ }^{5}$ \\ Wagner Anacleto Pinheiro ${ }^{6}$ \\ Ricardo Pondé Weber
}

\begin{abstract}
Resumo
O tecido de poliamida aromática (poliaramida) da marca Twaron foi reforçado por uma solução de óxido de grafeno para a produção de um nanocompósito, e posteriormente submetido a tratamento térmico. A impregnação do óxido de grafeno no tecido de poliaramida visa melhorar as propriedades balísticas do material por meio do aumento do coeficiente de atrito entre as fibras do tecido. Este estudo avaliou a adesão das folhas de óxido de grafeno nos tecidos de poliaramida, utilizando a análise por espectroscopia de infravermelho por transformada de Fourier (FTIR), a análise termogravimétrica (TGA) e a microscopia eletrônica por varredura (MEV), após o tratamento térmico. Por meio da análise dos resultados, foi possível observar que a adesão do óxido de grafeno aumentou com o tratamento térmico empregado neste trabalho.
\end{abstract}

Palavras-chave: Óxido de grafeno, poliamida aromática, nanocompósito, tratamento térmico.

\section{STUDY OF THE ADHESION OF GRAFENE OXIDE IN AN AROMATIC POLYAMIDE FABRIC BY THERMAL TREATMENT}

\begin{abstract}
The aromatic polyamide fabric (polyaramide) of the Twaron brand was reinforced by a solution of graphene oxide for a production of a nanocomposite, and subjected to a heat treatment. The impregnation of graphene oxide in polyaramide fabric is intended to improve the ballistic properties of the material by increasing the coefficient of friction between the fibers of the fabric. This study evaluated a treatment of graphene oxide sheets in polyaramide tissues using a Fourier Transform Infrastructure (FTIR), Thermogravimetric (TGA) and Scanning Electron Microscopy (SEM) analysis after heat treatment. By means of the analysis of the results, it was possible to observe that the adhesion of graphene oxide increased with the thermal treatment used in this work.
\end{abstract}

Keywords: Graphene oxide, aromatic polyamide, nanocomposite, heat treatment.

1 Licenciado em Química, mestrando em Ciência dos Materiais, Seção de Engenharia Mecânica e de Materiais, Instituto Militar de Engenharia, Rio de Janeiro, RJ - Brasil.

2 Engenheira Mecânica, doutoranda em Ciências dos Materiais, Instituto Militar de Engenharia, Rio de Janeiro, RJ - Brasil.

3 Engenheira Metalúrgica, mestranda em Ciências dos Materiais, Instituto Militar de Engenharia, Rio de Janeiro, RJ - Brasil. 
4 Tecnóloga em Polímeros, doutoranda em Ciências dos Materiais, Instituto Militar de Engenharia, Rio de Janeiro, $R J$ - Brasil.

5 Engenheira Química, doutora em Ciência e Tecnologia de Polímeros, professora titular da Universidade Federal do Rio de Janeiro, RJ - Brasil.

6 Engenheiro Metalúrgico, doutor em Ciências dos Materiais, professor do Instituto Militar de Engenharia, Rio de Janeiro, RJ - Brasil.

7 Engenheiro Mecânico, doutor em Ciências dos Materiais, professor e pesquisador do Instituto Militar de Engenharia, Rio de Janeiro, RJ - Brasil.

\section{INTRODUÇÃO}

A partir da década de 70 a utilização de tecidos para produção de compósitos e nanocompósitos tem tido grande relevância por conferirem excelentes propriedades a estes materiais. Dentre esses tecidos, destacam-se aqueles fabricados com fibra sintética de poliaramida, devido ao elevado desempenho como reforço em materiais compósitos com matriz polimérica (PCM's).

Estas fibras possuem excelentes propriedades tais como baixa densidade, alta resistência específica e tenacidade, o que as tornam extremamente adequadas para a produção de equipamentos de proteção balística, onde a mobilidade é fundamental no desempenho deste material [1].

Devido à superfície lisa e estrutura química inerte destas fibras, a adesão superficial entre as mesmas se torna fraca o que diminui algumas propriedades mecânicas e especialmente balísticas [2]. Como resultado, o estudo de diferentes tipos de tratamentos superficiais para aumentar a interação superficial e consequentemente suas propriedades mecânicas e balísticas se faz necessário.

Algumas pesquisas objetivam aumentar a tensão superficial desta fibra e consequentemente aumentar o atrito entre as mesmas, bem como entre os tecidos produzidos por tais fibras, melhorando desta forma a resistência balística [2]. Os métodos mais utilizados são: (a) tratamento com plasma (b) ativação química e (c) revestimento com agentes de acoplamento [1].

Estudos relacionados a tratamentos superficiais por enxerto de nanotubos de carbono (CNT's) na superfície das fibras de poliaramida [3] resultaram em um aumento de $30 \%$ na resistência ao cisalhamento interfacial de compósitos com matriz bismaleimida modificada, reforçada por tecidos deste material. Esta técnica permite uma melhora simultânea no desempenho mecânico e na tensão superficial da fibra de poliaramida. A aplicação de nanoplaquetas de grafeno na superfície destes tecidos, ao invés dos nanotubos de carbono, apresentou uma sensível melhora nas propriedades mecânicas do compósito [1,4] (resistência à tração, módulo de Young, tenacidade à fratura). Esta melhora é atribuída ao melhor encaixe mecânico na interface nano carga-matriz e à sua geometria bidimensional, sugerindo que o óxido de grafeno (GO) pode ser responsável pela maior rigidez obtida no compósito, resultante do arranjo hexagonal e bidimensional (2D) dos átomos de carbono ligados covalentemente em uma estrutura em configuração favo de mel [4], como apresentado na Figura 1.

Outros estudos [5,6] sugerem que o GO, por consistir de regiões intercaladas com carbonos hibridizados $\mathrm{sp}^{2}$, contendo grupos carboxila, e carbonos hibridizados $\mathrm{sp}^{3}$, com grupos funcionais hidroxila e epóxi em seus planos basais (Figura 2), aumentam a interação com as fibras poliméricas. 


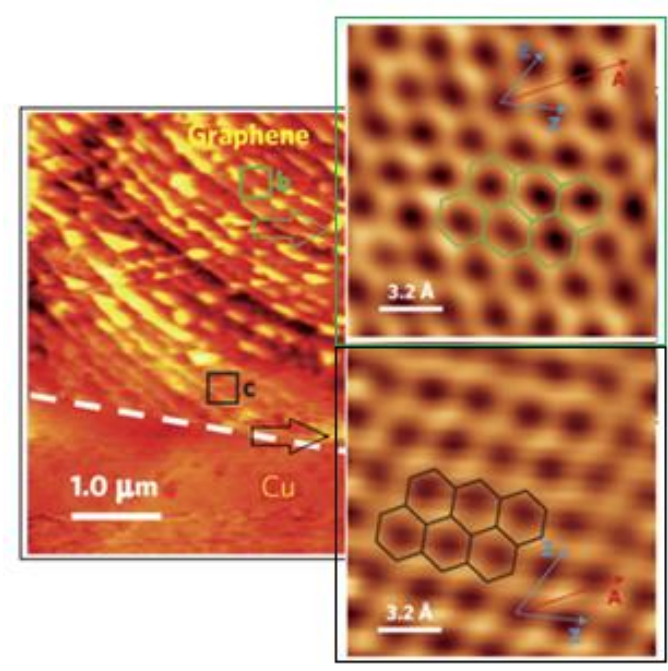

Figura 1. Estrutura em favo de mel de um grão de GO, obtido em Microscopia de Tunelamento de Varredura (STM) em um cristal de cobre; adaptado de Yu et al., 2011 [7].

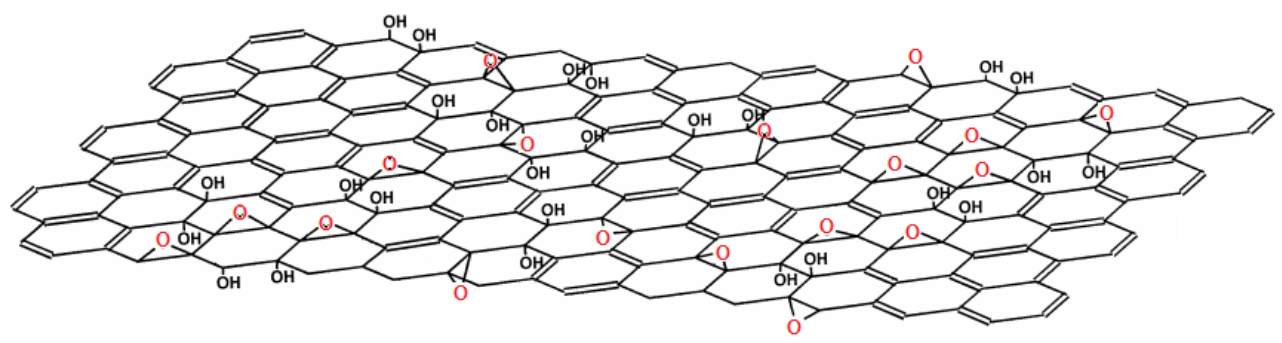

Figura 2. Estrutura química de uma única folha de GO, adaptado do modelo Lerf-Klinowski [8].

As propriedades elásticas de compósitos reforçados por fibras unidirecionais de poliaramida envelopados por uma fina camada de GO $[9,10,11]$ apresentaram um aumento no módulo de resistência de $60 \mathrm{GPa}$ para até $207 \mathrm{GPa}$ devido a melhor interação química entre o GO e as cadeias poliméricas.

Segundo Martirosyan e colaboradores [11], uma melhor compatibilidade interfacial é alcançada por meio da modificação de grupos funcionais (ácidos carboxílicos) ligados às superfícies. Assim, alterações na estrutura física ou química das fibras de poliaramida podem por meio de tratamento térmico proporcionar um aumento dos microcristais, quando aquecidos, o que possivelmente acarretaria no surgimento de microporos na superfície das fibras [12], e consequentemente, uma melhor adesão do GO às fibras de poliaramida.

Assim sendo, o presente trabalho visa avaliar a melhora na adesão interfacial do óxido de grafeno nas fibras de poliaramida devido a formação de possíveis grupos funcionais na sua superfície após tratamento térmico.

\section{MATERIAIS E MÉTODOS}

\subsection{Preparação das amostras de poliaramida e de óxido de grafeno}

Três amostras de poliaramida com diametro de $4 \mathrm{~cm}$ foram retiradas de um tecido da marca Twaron $\AA$, com trama T750 e gramatura $460 \mathrm{~g} / \mathrm{m}^{2}$, fornecido pela empresa Teijin 
Aramid, sob a forma de bobina do tipo 3360 dtex - 1000/1000. As amostras foram aquecidas a $80^{\circ} \mathrm{C}$ por 30 minutos, para remoção da umidade, e pesadas.

O óxido de grafeno foi obtido a partir do grafite Sigma-Aldrich 332461, por meio do método de Hummers modificado [13]. Basicamente, a partir de grafite em flocos são realizadas as seguintes etapas: (i) intercalação, por meio da adição de ácido sulfúrico e nitrato de sódio; (ii) oxidação, por meio da adição de permanganato de potássio, com agitação durante 7 dias; (iii) esfoliação, por meio de adições de soluções diluídas de ácido sulfúrico e peróxido de hidrogênio, e ciclos de lavagem e centrifugação. Assim, são geradas folhas de GO com monocamadas ou poucas camadas, como a esquematizada na Figura 2.

\subsection{Preparação das amostras de poliaramida impregnadas por óxido de grafeno}

Uma solução de $10 \mathrm{ml}$ de óxido de grafeno suspenso em meio aquoso, com concentração de $2 \mathrm{mg} / \mathrm{ml}$ foi preparada para ser depositada no tecido de poliaramida. Esta deposição foi realizada pelo método de filtragem a vácuo em ambas as superfícies do tecido.

Posteriormente, todas as amostras foram secas a vácuo utilizando um dessecador de vidro. Após esta secagem as amostras foram tratadas térmicamente a uma temperatura de $120^{\circ} \mathrm{C}$ durante 30 minutos em uma estufa. Por fim, as amostras foram novamente pesadas.

Para avaliar a adesão do GO na superfície do tecido foi realizada uma manipulação manual do tecido por cerca de 1 minuto, sendo em seguida observada no microscópio eletronico de varredura a amostra manipulada e não manipulada.

A Figura 3 apresenta a amostra antes e após a deposição do óxido de grafeno na superfície. Foram retirados das amostras 3 corpos de provas para análise físicoquímica dos materiais.
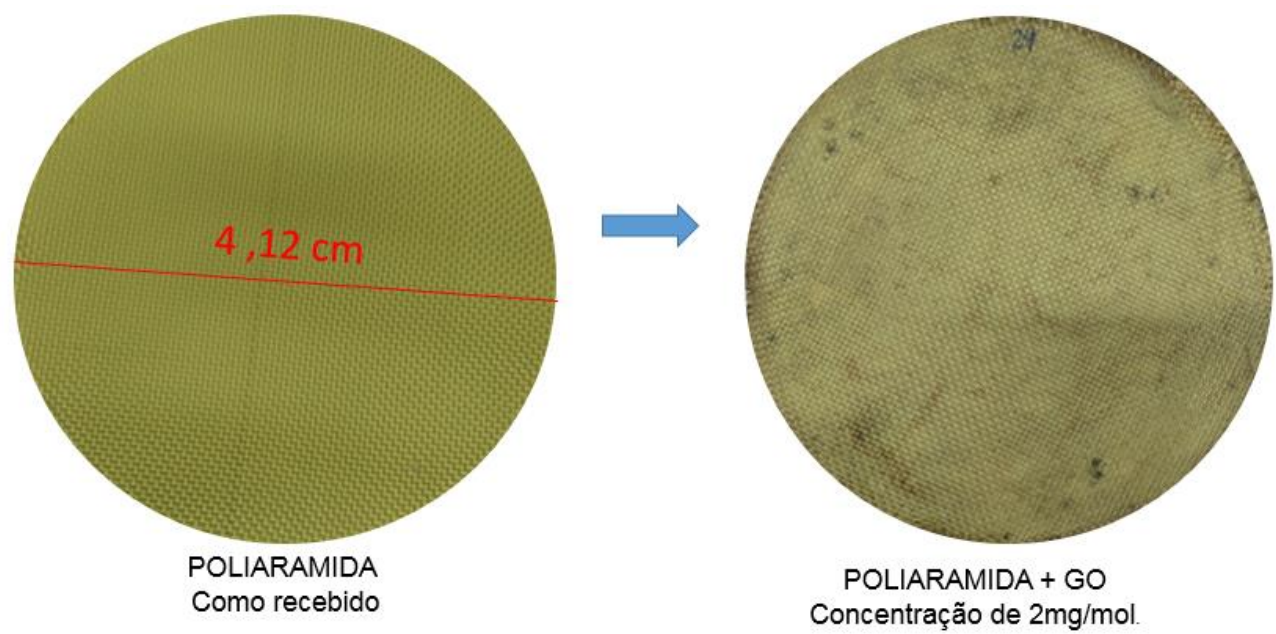

Figura 3. Amostras utilizadas para avaliação físico-química. Para preparação das amostras com GO foram utilizados $10 \mathrm{ml}$ de solução de GO a $2 \mathrm{mg} / \mathrm{ml}$.

\subsection{Análises físico-químicas}

A análise fisico-quimica foi realizada no tecido de poliaramida como recebido (CR), no tecido impregnado de GO e no GO, para termos de comparação. Os ensaios de 
espectroscopia de infravermelho por transformada de Fourier (FTIR) e a análise termogravimétrica (TGA) foram realizados no Laboratório de Apoio Instrumental (LAPINI) do Instituto de Macromolécula Eloísa Mano (IMA-UFRJ), visando caracterizar as mudanças na estrutura química da superfície e da composição das fibras modificadas, em relação ao material $\mathrm{CR}$, bem como no GO.

A espectroscopia FTIR foi realizada por meio de um espectrômetro modelo Frontier FT-IR/FIR, fabricante PerKin Elmer, na região entre $4000 \mathrm{~cm}^{-1}$ e $650 \mathrm{~cm}^{-1}$, empregando-se a técnica de refletância total atenuada (ATR). Os espectros em transmissão foram obtidos com resolução de $4 \mathrm{~cm}^{-1}$ e 60 varreduras em cada ensaio e processados em um programa gerenciador de dados.

A análise por FTIR foi utilizada para verificar a existência de alterações na estrutura das amostras estudadas, servindo como uma ferramenta para identificação de possíveis variações no comprimento de onda e absorções características das amostras. Os espectros foram normalizados a partir do pico a $822 \mathrm{~cm}^{-1}$, atribuída a vibrações de estiramento das ligações $\mathrm{C}-\mathrm{H}$ do anel aromático presente na estrutura principal da fibra de poliaramida utilizada para análise detalhada no intervalo 1750 $750 \mathrm{~cm}^{-1}$. Esta banda não apresenta variações significativas de intensidade quando a fibra é submetida a tratamento termo-químico [14,15].

A análise termogravimétrica (TGA) das amostras foram realizadas em um analisador termogravimétrico da marca TA Instruments, modelo TGA-Q500, dispondo de um par termoelétrico de cromel-alumel e de um sistema computadorizado de análise.

As amostras foram submetidas, sob ar, a um ciclo de aquecimento com a temperatura variando de $30^{\circ} \mathrm{C}$ até $800^{\circ} \mathrm{C}$, na velocidade de $10^{\circ} \mathrm{C} / \mathrm{min}$. Foram determinadas as temperaturas de início da perda de massa (onset).

\subsection{Análise fratográfica}

O exame fratográfico foi executado em um microscópio eletrônico de varredura, marca JEOL, modelo JSM 5800LV, utilizando-se energia do feixe de elétrons de 20 e $30 \mathrm{kV}$, observando-se a topografia das superfícies dos tecidos impregnados e como recebido com $1600 \times$ de magnitude e em baixo vácuo. O exame teve por finalidade verificar a morfologia superficial das amostras

\section{RESULTADOS E DISCUSSÃO}

\subsection{FTIR das fibras com adesão de GO}

A Figura 4 apresenta o espectro obtido para o tecido de poliaramida CR. Em relação ao espectro obtido, as principais bandas características de absorção da poliaramida estão relacionadas ao agrupamento amida e o anel aromático. Em $3013 \mathrm{~cm}^{-1}$ ocorre estiramento $\mathrm{N}-\mathrm{H}$, quando $\mathrm{O} \mathrm{C}=\mathrm{O}$ e $\mathrm{N}-\mathrm{H}$ encontram-se na configuração trans, caracterizando a amida $A$; em $1641 \mathrm{~cm}^{-1}$ tem-se a amida I, onde ocorre estiramento $\mathrm{C}=\mathrm{O}$; em $1537 \mathrm{~cm}^{-1}$ e $1302 \mathrm{~cm}^{-1}$ encontram-se respectivamente a amida II e III, sendo elas, a combinação do estiramento $\mathrm{C}-\mathrm{N}$ e flexão $\mathrm{N}-\mathrm{H}$; as bandas absorventes de grupos aromáticos (por exemplo, em $1500 \mathrm{~cm}^{-1}$ e $1390 \mathrm{~cm}^{-1}$ referenciadas a flexão no plano $\mathrm{C}-\mathrm{H}$; em $822 \mathrm{~cm}^{-1}$ observa-se flexão fora do plano $\mathrm{C}-\mathrm{H}$ ) também são observadas no tecido CR $[16 ; 17]$. 


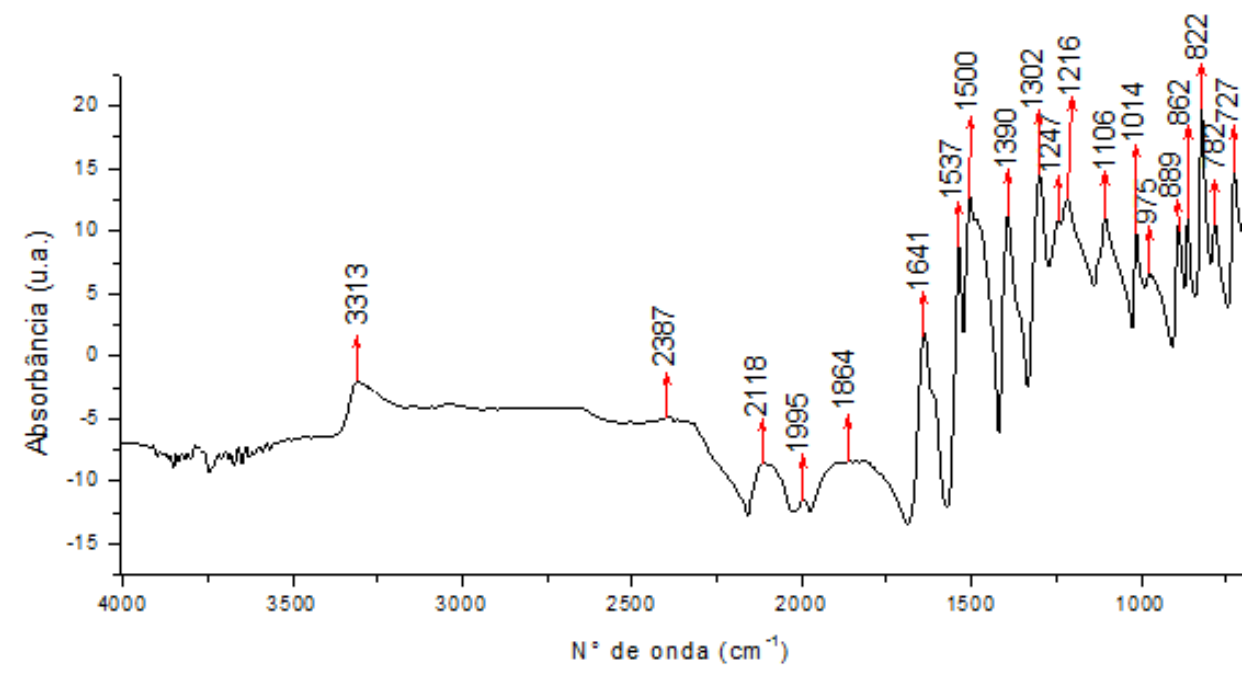

Figura 4. Espectro obtido de FTIR em modo absorbância para o tecido de poliaramida CR.

As alterações produzidas pela deposição do óxido de grafeno foram estudadas por comparação ao espectro do tecido de poliaramida CR. Estas estão apresentadas na superposição dos espectros na Figura 5 a.

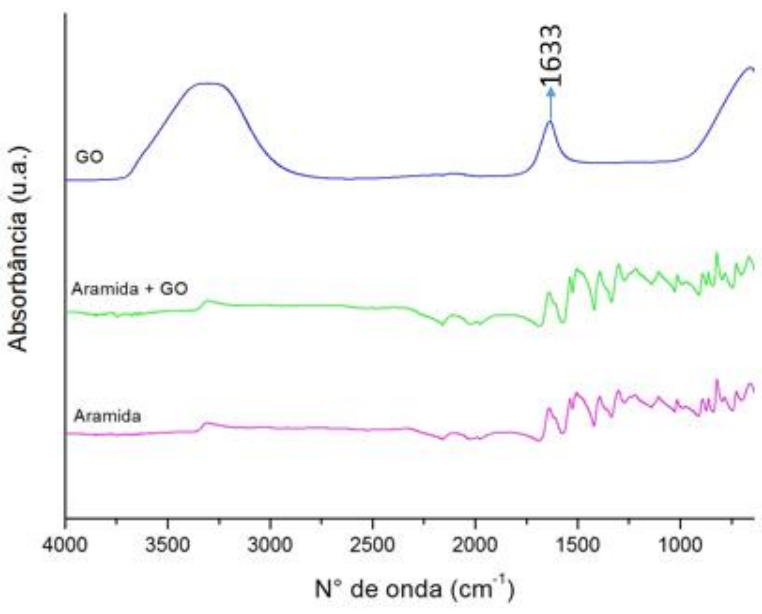

a)

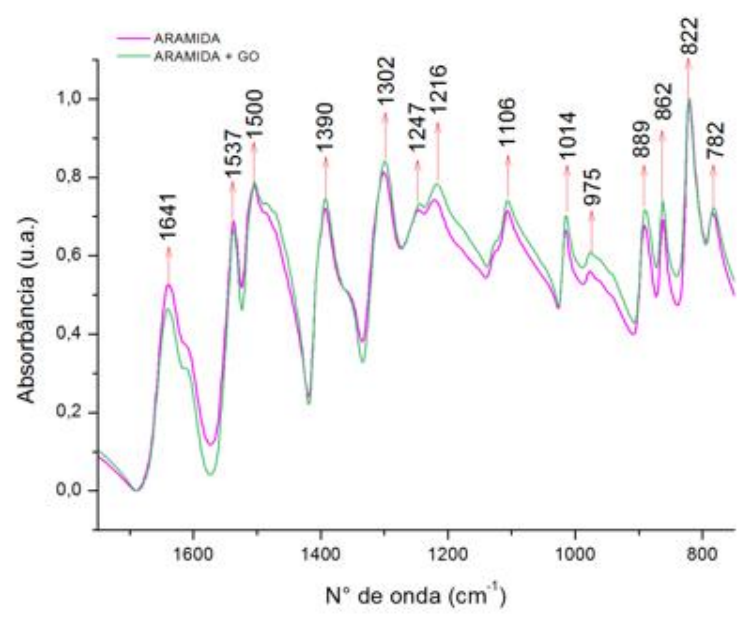

b)

Figura 5. Análises de FTIR na fibra de poliaramida antes e após deposição de GO. a) Espectros da poliaramida, poliaramida com GO e GO; b) Espectros normalizados pela banda de $822 \mathrm{~cm}^{-1}$.

A Figura 5.b apresenta os espectros normalizados. Observa-se nesta figura que a intensidade nas principais bandas varia após deposição do óxido de grafeno. A partir da análise dos espectros normalizados, verifica-se que a contribuição do pico carbonila do GO [14] (estiramento $\mathrm{C}=\mathrm{O}$ em $1633 \mathrm{~cm}^{-1}$ ) gerou uma diminuição na intensidade da banda $1641 \mathrm{~cm}^{-1}$ do nanocompósito, indicando que as vibrações do estiramento $\mathrm{C}=\mathrm{O}$ encurtaram os grupos funcionais $-\mathrm{COOH}$, ligados às superfícies da fibra modificada [1].

Também, pode-se notar uma ligeira diminuição na intensidade do pico de flexão N-H e C-H (na banda $1537 \mathrm{~cm}^{-1}$ ), podendo ser atribuída a uma possível decomposição do grupo amida. Este resultado sugere que o aumento dos grupos $\mathrm{C}=\mathrm{O}(\mathrm{e} \mathrm{O}=\mathrm{C}-\mathrm{O})$ pode resultar na clivagem da ligação C-N nos grupos aminas, além da oxidação na cadeia dos grupos terminais após o tratamento [14]. 
Contudo, os picos abaixo de $1500 \mathrm{~cm}^{-1}$ aumentam ligeiramente sua intensidade, o que pode ser atribuído às mudanças estruturais, como das folhas de óxido de grafeno quimicamente ligadas às fibras de poliaramida [1].

Outro possível meio pode ser devido ao estiramento de grupos $\mathrm{C}=\mathrm{C}$ no anel benzeno, que pode ser devido aos efeitos anteriormente citados (cisão dos grupos amida), além da conformação da cadeia do benzeno que é absorvido pelas flexões das bandas C$\mathrm{H}\left(1390 \mathrm{~cm}^{-1}-862 \mathrm{~cm}^{-1}\right)$ na superfície da fibra de aramida durante o tratamento [17]. E por fim, devido a solução de GO utilizada na pesquisa apresentar $\mathrm{pH} 4$, os resultados de uma adesão parcial de folhas GO na poliaramida são devido a deposição feita por banho de reação alcalina [1]. O que não aconteceria caso essa adesão fosse feita em reações ácidas que, por sua vez, reteriam maior número de grupos funcionais $-\mathrm{COOH}$, aumentando assim a compatibilidade interfacial dos materiais $[1,11]$.

\subsection{TGA das fibras com adesão de GO}

As curvas termogravimétricas das amostras de poliaramida, poliaramida + GO com tratamento térmico e GO são mostradas na Figura 6.

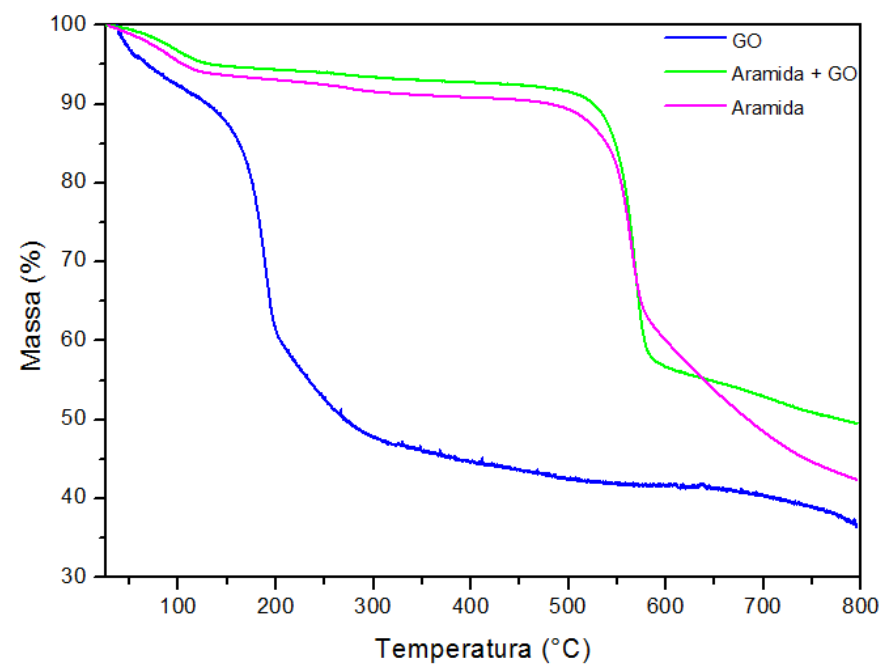

Figura 6. Análise termogravimétrica das amostras poliaramida, poliaramida + GO e GO.

Observa-se que em ambas as amostras de poliaramida ocorreram dois estágios referentes a perda de massa, indicadas pelos pontos de inflexão. O GO, contudo, apresentou três estágios de perda de massa.

Ao comparar as amostras (de poliaramida CR e poliaramida $+\mathrm{GO}$ ), percebe-se que no primeiro estágio, entre 27,5 e $121,9^{\circ} \mathrm{C}$; e 23,3 e $136,4^{\circ} \mathrm{C}$, as amostras de poliaramida (CR) e poliaramida + GO apresentaram pouca perda de massa, em torno de $6 \%$ e $5 \%$ respectivamente, resultado da eliminação de água [16]. A amostra de GO, também apresentou cerca de $8 \%$ (entre 38 e $93^{\circ} \mathrm{C}$ ) de perda de massa associada à eliminação de água [18].

Analisando o processo de decomposição do tecido de fibra de poliaramida como recebido e impregnado com GO, observa-se uma menor perda de massa no tecido impregnado em torno de $52 \%$, na temperatura entre 473 e $578^{\circ} \mathrm{C}$, provavelmente associada a formação de resíduos compostos por anéis aromáticos, quando comparada a perda de massa de $56 \%$ do tecido de poliaramida CR, associada a quebra da cadeia macromolecular e pirólise dos grupos funcionais [16]. 
Em relação ao $\mathrm{GO}$, é observada uma perda de $30 \%$ de massa em $200^{\circ} \mathrm{C}$ que se refere à eliminação de grupos funcionais oxigenados menos estáveis presentes nas folhas do mesmo [18] e [19].

Por fim, observa-se mais uma perda de massa no $\mathrm{GO}$ entre $202^{\circ} \mathrm{C}$ até a região de $792^{\circ} \mathrm{C}$, que está relacionado à perda de massa por meio da remoção de grupos funcionais mais estáveis [18].

Observa-se desta forma que o tecido de poliaramida impregnado com GO e tratado termicamente possui uma maior estabilidade quando comparado ao tecido como recebido, indicando uma maior adesão do GO à poliaramida.

\subsection{Morfologia superficial do tecido de fibra de poliaramida impregnada e como recebida}

A Figura 7 apresenta a morfologia da superfície da fibra de poliaramida como recebida, impregnada e tratada termicamente, bem como manipulada
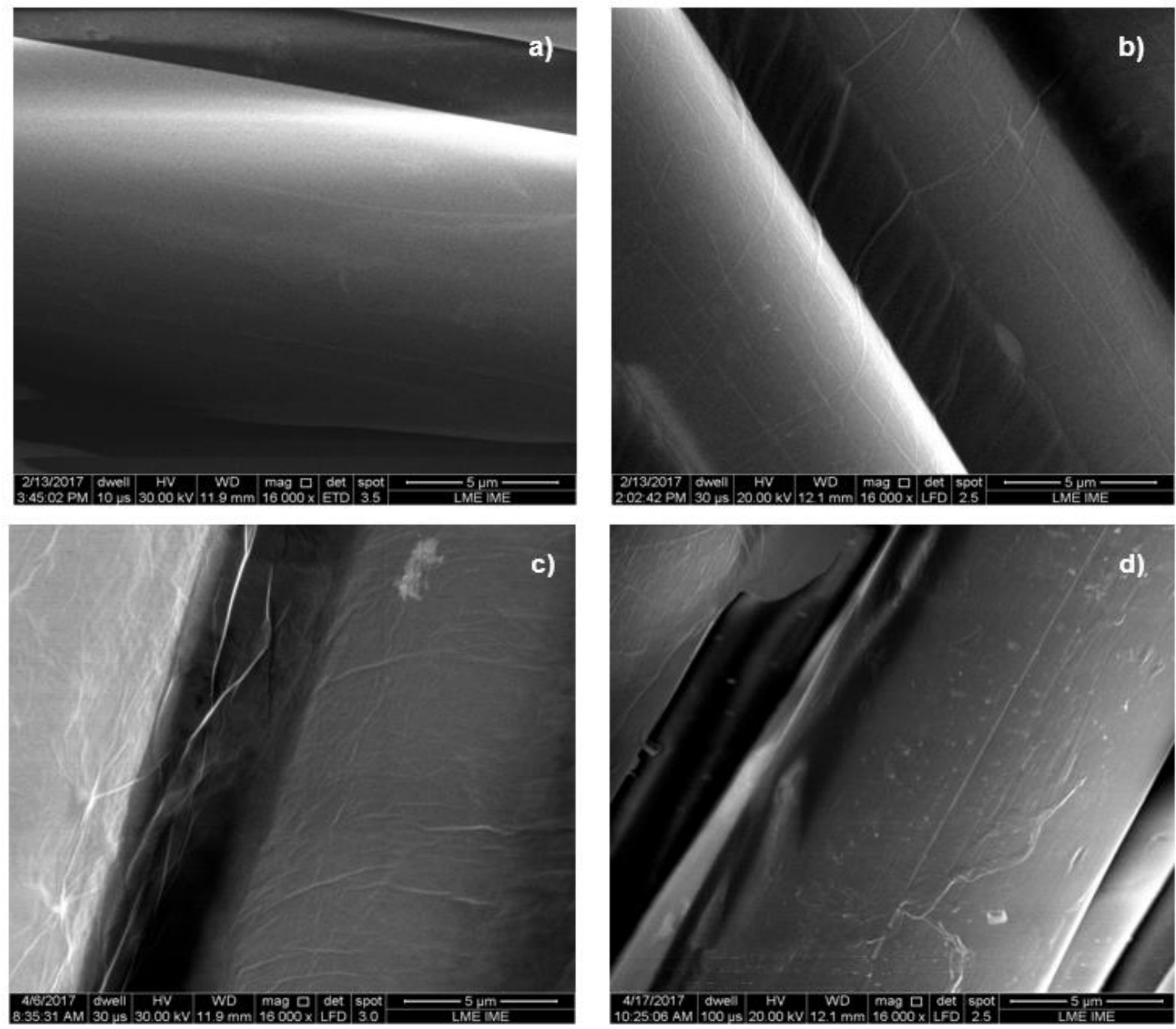

Figura 7. Comportamento da impregnação das folhas de GO na superfície da fibra de poliaramida. a) Fibras de poliaramida CR; b) fibras com deposição de GO/concentração de $2 \mathrm{mg} / \mathrm{mol}$; c) fibras com GO após tratamento térmico $120^{\circ} \mathrm{C}$; d) fibras de poliaramida manipuladas após tratamento. 
A Figura 7.a apresenta a estrutura lisa da fibra de poliaramida como recebida. $\mathrm{Na}$ Figura 7.b observa-se a distribuição homogênea das folhas de GO na superfície do tecido de poliaramida impregnado com GO.

A Figura 7.c apresenta a adesão uniforme do GO na fibra de poliaramida após tratamento térmico realizado. Pode-se notar que as folhas de GO se apresentam contraídas na superfície da fibra, provavelmente pela eliminação da agua do GO. A Figura 7.d apresenta uma micrografia do tecido de fibra de poliaramida impregnada por GO após manipulação. Observa-se que apesar da quebra parcial das folhas de GO, umas quantidades significativas ainda se mantêm aderida a fibra, possivelmente devido ao surgimento da alta densidade dos grupos funcionais nas áreas superficiais da fibra [15].

\section{CONCLUSÃO}

Os resultados sugerem que o tratamento térmico no tecido impregnado promove um aumento na adesão do óxido de grafeno à fibra. Este aumento na adesão está provavelmente relacionado à formação dos grupos funcionais $(-\mathrm{COOH})$ na superfície da fibra e a maior interação destes com os grupos oxigenados no óxido de grafeno, como observado pelas análises de FTIR e TGA. A análise morfológica por MEV confirma este resultado, apontando para uma maior homogeneização do óxido de grafeno na superfície da fibra após o tratamento térmico realizado, o que sugere uma adesão superficial mais homogênea.

\section{Agradecimentos}

Os autores agradecem ao Laboratório de Microscopia Eletrônica (IME) e ao Laboratório de Apoio Instrumental (IMA-UFRJ) pela contribuição nas análises, e à Coordenação de Aperfeiçoamento de Pessoal de Nível Superior (CAPES) pelo apoio financeiro.

\section{REFERÊNCIAS}

1 Hussain, S., Yorucu, C., Ahmed, I., Hussain, R., Chen, B., Khan, M.B., Siddique, N.A., Rehman, I. Ur. Surface modification of aramid fibres by graphene oxide nano-sheets for multiscale polymer composites. Surface and Coatings Technology. 2014; 258: 458-466. Jia, C., Chen, P., Liu, W., Li, B., Wang, Q. Surface treatment of aramid fiber by air dielectric barrier discharge plasma at atmospheric pressure. Applied Surface Sience. 2011. 257:4165-4170.

3 Chen, W., Qian, X.-M., He, X.-Q., Liu, Z.-Y., Liu, J.-P. Surface modification of Kevlar by grafting carbon nanotubes. 2012. Journal of Applied Polymer Science, 123: 1983-1990.

4 Lee, J.-H., Loya, P.E., Lou, J., Thomas, E.L. Dynamic mechanical behavior of multilayer graphene via supersonic projectile penetration. Science. 2014; 346 (6213): 1092-1096.

5 Lee, D. W., De Los Santos L. V., Seo, J. W., Felix, L. L., Bustamante, A. D., Cole, J. M., Barnes, C. H. W. The structure of graphite oxide: Investigation of its surface chemical groups. 2010; arXiv preprint arXiv:1008.0937.

6 Szabó, T., Berkesi, O., \& Dékány, I. DRIFT study of deuterium-exchanged graphite oxide. Carbon, 2005; 43(15): 3186-3189.

7 Yu, Q., Jaregui, L.A., Wu, W., Colby, R., Tian, J., Su, Z. et al. Control and characterization of individual grains and grain boundaries in graphene grown by chemical vapour deposition. Nature Materials. 2011; 10: 443-449.

$8 \mathrm{He}, \mathrm{H}$., Klinowski, J., Forster M., Lerf, Anton. A new structural model for graphite oxide. Chemical Physics Letters. 1998; 287: 53-56. 
9 Mukhopadhyay, P., Gupta, R.K. Graphite, Graphene, and their Polymers Nanocomposites. CRC Press. 2013: 589 p.

10 Martirosyan, K. S., Zyskin, M. Simulation of the Elastic Properties of Reinforced KevlarGraphene Composites. Int. J. Nanosci. 2012; 11(3): 1-11.

11 Martirosyan, K.S., Zyskin, M. Modeling and Simulation of the Elastic Properties of Kevlar Reinforced by Graphene. Cap.2 . 19-25. By Graphene Science Handbook: SizaDependet Properties. Aliofkhazraei, M., Ali, N., Milne, W. I., Ozkan, C.S., Mitura, S., Gervasoni J.L. CRC Press. 2016: 505 p.

12 Yue, C.H., Sui, G.X., Looi, H.C. Effects of heat treatment on the mechanical properties of Kevlar-29 fibre. Composites Science and Technology. 2000; 60: 412-427.

13 Rourke, J. P., Pandey, P. A., Moore, J. J., Bates, M., Kinloch, I. A., Young, R. J. and Wilson, N. R. The Real Graphene Oxide Revealed - Stripping the Oxidative Debris from the Graphene-like Sheets. Angew. Chem. Int. Ed. 50, $3173-3177,2011$.

14 Jia, C., Chen, P., Li, N., Wang, Q., Lu, C., Yu, Q. Effects of Twaron fiber surface treatment by air dielectric barrier discharge plasma on the interfacial adhesion in fiber reinforced composites. Surface \& Coatings Technology. 2010; 204: 36683675.

15 Rodríguez-Uicab, O., Avilés, F., Gonzalez-Chi, P.I., Canché-Escamilla, G., Duarte-Aranda, S., Yazdani-Pedram, M., Toro, P., Gamboa, F., Mazo, M.A., Nistal, A., Rubio, J. Deposition of carbon nanotubes onto aramid fibers using asreicevedand chemically modified fibers. Applied Surface Science. 2016; 385: 379-390.

16 Nascimento, R. F., Miguez Suarez, J.C., Weber, R.P. Influência do intemperismo no desempenho mecânico e balístico de tecido produzido com fibra de aramida. 2015. Dissertação de Mestrado - Instituto Militar de Engenharia; Rio de Janeiro, 2015: 141 p.

17 Zhang, Y.H., Zhang, J., Chen, J., Hao, X., Wang, S., Feng, X., Guo, Y. Effects of solar UV irradiation on the tensile properties and structure of PPTA fiber. Polymer Degradation and Stability. 2006; $91: 2761-2767$.

18 Chen, J., Yao, B. Li, C., Shi, G. An improved Hummers method for eco-friendly synthesis of graphene oxide. Carbon. 2013; 64: 225-229.

19 Paredes, J. I. Villar-Rodil, S., Martínez-Alonso, A. Tascón, J. M. D. Graphene Oxide Dispersions in Organic Solvents. Langmuir. 2008; 24: 10560-10564. 